\title{
Algebraic connectivity optimization via link addition
}

\author{
Huijuan Wang and Piet Van Mieghem \\ Delft University of Technology, P.O. Box 5031, 2600 GA Delft, The Netherlands \\ Email: $\{$ H.Wang, P.F.A.VanMieghem\}@tudelft.nl
}

\begin{abstract}
Significant research effort has been devoted to the topological features of complex networks to enhance the performance of dynamic processes implemented on these networks. In this work, we investigate how to optimize a network for a given dynamic process via a minor topological modification. The algebraic connectivity $a(G)$ of a network $G$ characterizes the performance of e.g. synchronization of dynamic processes at the nodes of the network $G$ and random walks on networks which models e.g. the dispersion phenomena. We confine ourselves to the problem: "Where to add a link in a network $G$ such that the algebraic connectivity is increased the most?" Exhaustive searching for the optimal link addition is computationally infeasible. Hence, we propose two strategies: 1 . adding a link between the minimal degree node and a random other node; 2 . adding a link between a node pair with the maximal $\left|u_{i}-u_{j}\right|$, the absolute difference between the $i-t h$ and $j-t h$ elements of the Fiedler vector of $G$. Strategy 1 and 2 are compared with random link addition in three classes of networks: the Erdös-Rényi random graph, the BA model and the $k$-ary tree. The Fiedler vector based strategy 2 performs better than strategy 1 . However, strategy 1 requires only local information, i.e. node degree.
\end{abstract}

\section{Keywords}

Algebraic connectivity, synchronization, optimization, link addition

\section{INTRODUCTION}

Topologies of complex networks ranging from biological networks such as gene regulatory networks [1], metabolic networks[2], artificial networks like the Internet, the WWW to social networks, e.g. paper citations, collaboration networks etc. [3], have been accumulated by active investigation in recent years. Significant research efforts have been conducted in their topological features and the corresponding network modeling [4][5]. Researchers from physics, bi-

Permission to make digital or hard copies of all or part of this work for personal or classroom use is granted without fee provided that copies are not made or distributed for profit or commercial advantage and that copies bear this notice and the full citation on the first page. To copy otherwise, to republish, to post on servers or to redistribute to lists, requires prior specific permission and/or a fee.

Bionetics '08, November 25-28, 2008, Hyogo, Japan

Copyright 2008 ICST 978-963-9799-35-6. ology, psychology, sociology and economics have been also actively involved in the performance analysis of dynamic processes on complex networks, e.g. diffusion of information and synchronization processes on e.g. neural networks. In this work, we explore the following question: How to optimize a network for a certain dynamic process via a minor topology modification?

The algebraic connectivity $a(G)$ or the second smallest eigenvalue of the Laplacian matrix of a network $G$, defined in Section 2, is widely studied in the literature due to (a) its importance for the connectivity, a basic measure of the robustness of a graph. The larger the algebraic connectivity is, the larger the relative number of links required to be cutaway to generate a bipartition [6]; (b) its correlation with performance of dynamic processes, e.g. synchronization of dynamic processes at the nodes of a network and random walks on graphs which model e.g. the dispersion phenomena or exploring graph properties [6]. A network has a more robust synchronized state ${ }^{1}$ if the algebraic connectivity of the network is large $[7,8]$. Random walks move and disseminate efficiently in topologies with large algebraic connectivity.

In this paper, we confine our problem as the following: "where should we add a link to a network $G$ such that the algebraic connectivity $a(G)$ can be increased the most?" For large real-world networks, to improve the network performance, a minor modification on the current network, i.e. adding a small number of links, is usually required due to economic concerns. The investigation on adding one link to improve the algebraic connectivity will also provide insights on how to dynamically add a set of links one by one so that the algebraic connectivity is maximally increased. The number of possibilities of adding a link to a network $G(N, L)$ with $N$ nodes and $L$ links is $\left(\begin{array}{c}N \\ 2\end{array}\right)-L$. For large realistic (hence sparse) networks, it is infeasible to compare all these possibilities and find the optimal one. Hence, we propose two strategies of adding a link to optimize the algebraic connectivity. The node pair $(i, j)$ where a link can be added, can be characterized by various topological metrics such as the node degree, i.e. the number of links that is connected with the node, or the distance between the node pair. We propose two strategies based on these topological metrics. Strategies are compared together with random link addition in various classes of networks.

The structure of this paper is outlined as follows. Section 2 introduces the definition of algebraic connectivity as well as related theoretical results. The design of strategies are

\footnotetext{
${ }^{1}$ In human brain networks, a high synchronization should be avoided because it may cause epileptic attachs.
} 
explained in Section 3. We evaluate these strategies in Section 4 by comparing them with the random link addition. We conclude our paper in Section 5.

\section{BASIC DEFINITION AND THEORY}

Let $G$ be a graph and let $\mathcal{N}$ denote the set of nodes and $\mathcal{L}$ the set of links, with $N=|\mathcal{N}|$ nodes and $L=|\mathcal{L}|$ links, respectively. The Laplacian matrix of $G$ with $N$ nodes is an $N \times N$ matrix $Q=\Delta-A$, where $\Delta=\operatorname{diag}\left(d_{i}\right)$ and $d_{i}$ is the degree of node $i \in \mathcal{N}$ and $A$ is the adjacency matrix of $G$. The Laplacian eigenvalues are all real and nonnegative [9]. The set of all $N$ Laplacian eigenvalues $\mu_{N}=0 \leq \mu_{N-1} \leq$ $\ldots \leq \mu_{1}$ is called the Laplacian spectrum of $G$. Fiedler [10] showed that the second smallest eigenvalue $\mu_{N-1}$ is 0 if and only if the graph is disconnected. In addition, he proposed to call the second smallest eigenvalue of $Q$ as the algebraic connectivity of $G$ and it is denoted as $\mu_{N-1}=a(G)$ for simplicity. [11])

The following Theorem is well known (see, for example

Theorem 1. Let $\mathrm{G}$ be a general graph of $N$ nodes. Let $G+e$ be the graph obtained by adding a link $e$ between two nodes that are not directly connected in $G$. Then the eigenvalues of $G$ interlace those of $G+e$, that is,

$$
\begin{aligned}
0 & =\mu_{N}(G)=\mu_{N}(G+e) \leq \mu_{N-1}(G) \leq \mu_{N-1}(G+e) \\
& \leq \mu_{N-2}(G) \ldots \leq \mu_{1}(G) \leq \mu_{1}(G+e)
\end{aligned}
$$

It follows from Theorem 1 that, if the multiplicity of $\mu_{N-1}(G)$ is larger than 1, i.e. $\mu_{N-2}(G)=\mu_{N-1}(G)$, the algebraic connectivity remains the same after a link is added, i.e. $\mu_{N-1}(G)=\mu_{N-1}(G+e)$. Since $\operatorname{trace}(Q)=\sum_{i=1}^{N} \mu_{i}(G)=$ $2 L$. We deduce that

$$
\sum_{i=1}^{N}\left(\mu_{i}(G+e)-\mu_{i}(G)\right)=2
$$

which implies that at least one inequality in Theorem 1 must be strict and

$$
0 \leq \mu_{N-1}(G+e)-\mu_{N-1}(G) \leq 2
$$

When is the algebraic connectivity increased the most (i.e. by 2) via adding a link? According to [12],

Theorem 2. Let $G$ be a connected graph with $N$ nodes and let $i$ and $j$ be two non-adjacent nodes in $G$. The largest possible increase in the algebraic connectivity, i.e. $\mu_{N-1}(G+$ $e)=\mu_{N-1}(G)+2$ occurs if and only if $G=K_{N} \backslash\{i, j\}$, the complete graph minus one link.

Das [13] has proved the following theorem regarding to the pendants in a graph, which are nodes with degree 1.

Theorem 3. Let $G$ be a simple connected graph with $N>$ 2 nodes. If $G$ has a pendant node, then the algebraic connectivity is less than or equal to 1 . Moreover, the algebraic connectivity is strictly less than 1 if the pendant node is not adjacent to the highest degree node.

Real networks usually possess a set of pendants. Hence, the algebraic connectivity of a real-world network is likely to be smaller than $1, \mu_{N-1}(G)<1$, even after a link is added in the network.

We mention still another theorem of Das [13]:
Theorem 4. If an isolated node is connected by links to all the $N$ nodes of a graph $G$, then the Laplacian eigenvalues of the resultant graph are as follows: one of the eigenvalue is $N+1$, the other eigenvalues can be obtained by increasing the eigenvalues of the old graph $G$ by 1 except the smallest one 0 , which is always an eigenvalue of $Q$.

\section{STRATEGIES OF ADDING A LINK TO OBTAIN HIGH ALGEBRAIC CONNEC- TIVITY}

In this section, we investigate the increase of the algebraic connectivity due to the addition of a link between $\{i, j\}$ in relation with the topological characteristics of $\{i, j\}$. We propose link addition strategies based on topological metrics of $\{i, j\}$. The eigenvector based strategy is studied separately.

\subsection{Topological metrics based strategy}

Since $\mu_{N-1}(G) \leq \mu_{N-1}(G+e) \leq \mu_{N-2}(G)$ in Theorem 1 , we define the normalized increase of the algebraic connectivity as $0 \leq \mu^{*}=\frac{\mu_{N-1}(G+e)-\mu_{N-1}(G)}{\mu_{N-2}(G)-\mu_{N-1}(G)} \leq 1$. A link $e=(i, j)$ can be added if $i$ and $j$ are not yet connected in $G$. We start with a specific network to explore all possible realizations of adding a link to a graph in order to gain some insights in the relationship between $\mu^{*}$ and the topological characteristics of $\{i, j\}$ such as:

- degree of node $i$ and $j$;

- clustering coefficient of $i$ and $j$ : the clustering coefficient of a node $c_{G}(v)$ characterizes the density of connections in the environment of a node $v$ and is defined as the ratio of the number of links $y$ connecting the $d_{v}>1$ neighbors of $v$ over the total possible $\frac{d_{v}\left(d_{v}-1\right)}{2}$, thus $c_{G}(v)=\frac{2 y}{d_{v}\left(d_{v}-1\right)}$. We assume $c_{G}(v)=0$ if $d_{v}=1$.

- node betweenness of $i$ and $j$ : the betweenness $B_{v}$ of node $v$ is $B_{v}=\sum_{s \neq v \neq t \in \mathcal{N}, s \neq t} \frac{\sigma_{s t}(v)}{\sigma_{s t}}$, where $\sigma_{s t}$ is the number of shortest paths from $s$ to $t$, and $\sigma_{s t}(v)$ is the number of shortest paths from $s$ to $t$ that pass through node $v$.

- hopcount between node $i$ and $j$ : the number of hops or links in the shortest path between $i$ and $j$.

The correlation between $\mu^{*}$ introduced by the addition of link $(i, j)$ and topological metrics of the node pair $\{i, j\}$ is supposed to be topology dependent. We consider the following topologies: the Erdös-Rényi random graph $G_{p}(N)$ and the Barabási-Albert (BA) power law graph [4]. Traditionally, complex networks have been modeled as Erdös-Rényi random graphs $G_{p}(N)$, which can be generated from a set of $N$ nodes by randomly assigning a link with probability $p$ to each pair of nodes. Besides their analytic tractability, the Erdös-Rényi random graphs are reasonably accurate models for peer-to-peer networks and ad-hoc networks. The BA power law graph we generated starts with $m$ nodes; at every time step we add a new node with $m$ links that connect the new node to $m$ different nodes already present in the system. The probability that a new node will be connected to node $i$ is proportional to the degree $d_{i}$ of that node, i.e. 
$d_{i} / \sum_{j} d_{j}=d_{i} / 2 L_{t}$, where $L_{t}$ is the number of links in step $t$. The power law degree distribution is followed by many natural and artificial networks such as the scientific collaborations [14], the world-wide web, protein networks and the Internet [15].

\subsubsection{Erdös-Rényi random graph $G_{p}(N)$}

Given one Erdös-Rényi random graph $G=G_{0.6}(100)$, in each realization, a link $e=(i, j)$ is added to $G$ provided it does not exist in $G$, and we calculate $\mu^{*}$ and topological metrics of $\{i, j\}$. All possible realizations of adding a link to $G$ are considered. Figure 1(a) shows that $\mu^{*}$ is large if and

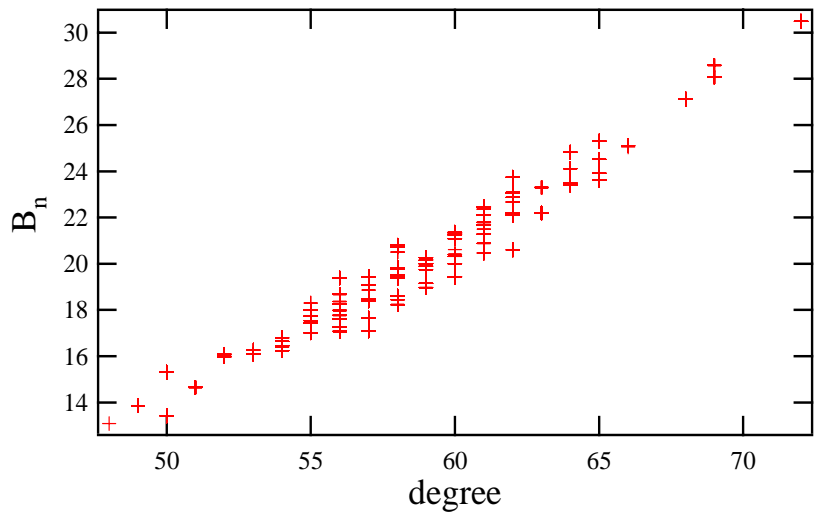

Figure 2: The degree and the node betweenness of a node in an Erdös-Rényi random graph $G_{0.6}(100)$.

only if one node of the pair $\{i, j\}$ has small degree. A similar correlation between $\mu^{*}$ and the node betweenness is observed in Figure 1(c). Furthermore, Figure 2 illustrates that the node betweenness is positively correlated with the degree of the node. Hence, the metric "degree" will be considered instead of the node betweenness whose computational complexity is much higher. The correlation between $\mu^{*}$ and the clustering coefficient is relatively weak as illustrated in Figure 1(b). There exists hardly correlation between $\mu^{*}$ and the hopcount between $i$ and $j$, which is mostly equal to 2 in such a dense graph.

\subsubsection{BA power law graph}

We perform the same experiment in a BA power law graph with $N=100$ and $m=3$. A small $m$ is selected such that the power law degree distribution can be observed for $N=100$. As shown in Figure 3, different from an ErdösRényi random graph, an relative high increase in $\mu^{*}$ implies low degree of both node $i$ and $j$. However, the inverse does not hold. Adding a link to two low degree nodes only modestly increases $\mu^{*}$. In fact, many nodes in a BA power law graph possesses a low degree. Again, the positive correlation between betweenness and degree of a node as shown in Figure 4, persuades us to consider the node degree which is simpler to compute than the node betweenness. Similarly, no clear correlation between $\mu^{*}$ and the clustering coefficient as well as the hopcount between $i$ and $j$ appear in Figure 3 (b) and 5 .

In view of the correlation between $\mu^{*}$ and the topological metrics regarding to node $i$ and $j$ where a link is added

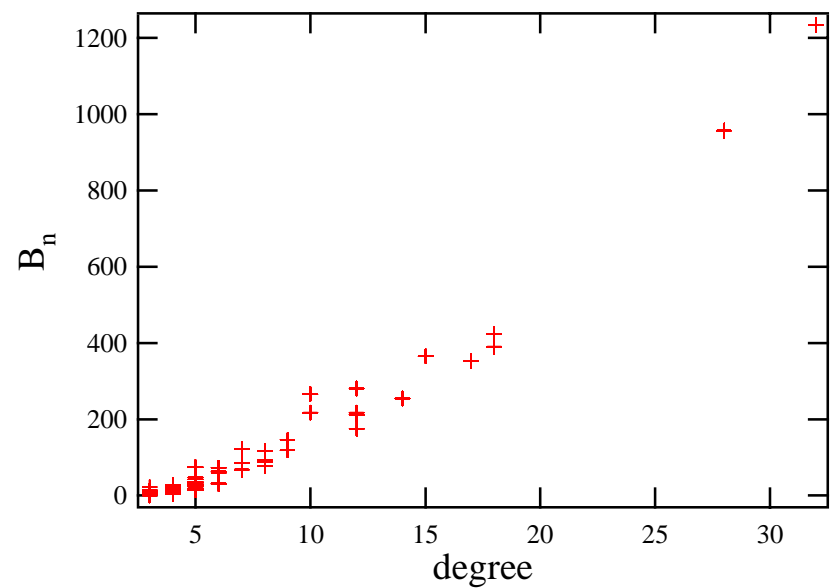

Figure 4: The degree and the node betweenness of a node in a BA model with $N=100$ and $m=3$.

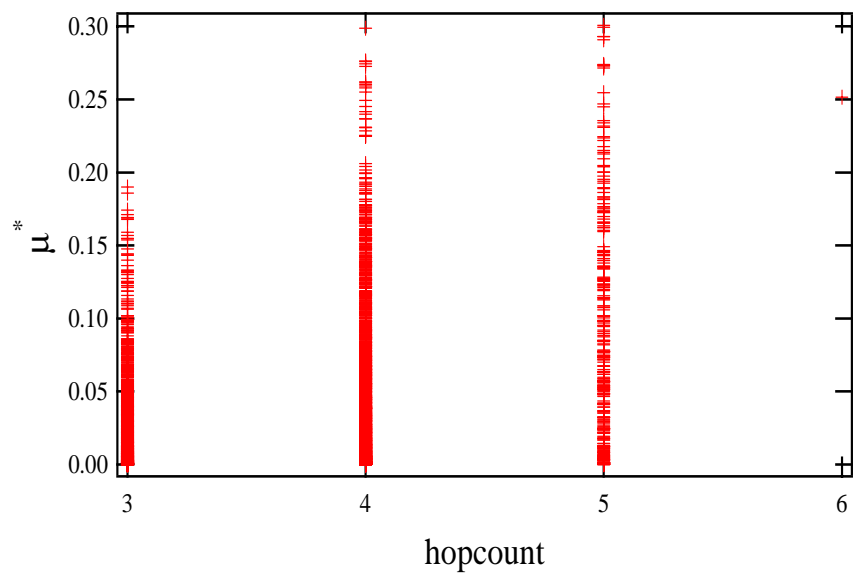

Figure 5: The increase of the algebraic connectivity $\mu^{*}$ due to the addition of link $(i, j)$ in relation with the hopcount between $i$ and $j$ in a BA model with $N=100$ and $m=3$. 


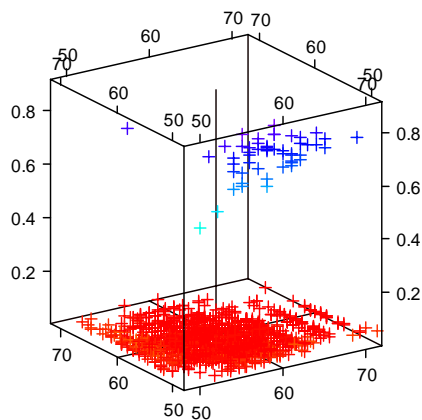

(a)

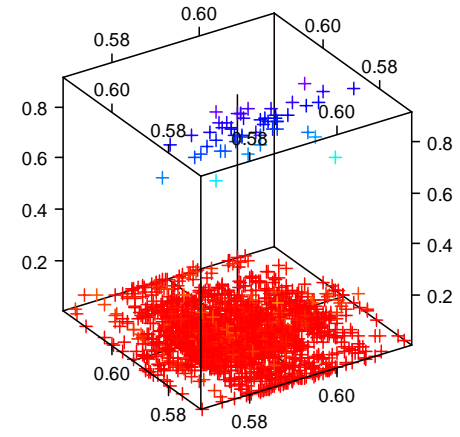

(b)

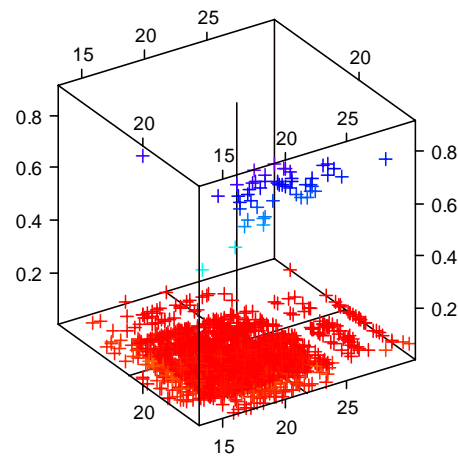

(c)

Figure 1: The increase of the algebraic connectivity $\mu^{*}$ (the $\mathrm{z}$ axis) due to the addition of link $\{i, j\}$ in relation with (a) the degree of node $i$ and $j$; (b) the clustering coefficient of node $i$ and $j$ (c) the betweenness of node $i$ and $j$ separately in $\mathrm{x}$ and y axis in an Erdös-Rényi random graph $G_{0.6}(100)$.

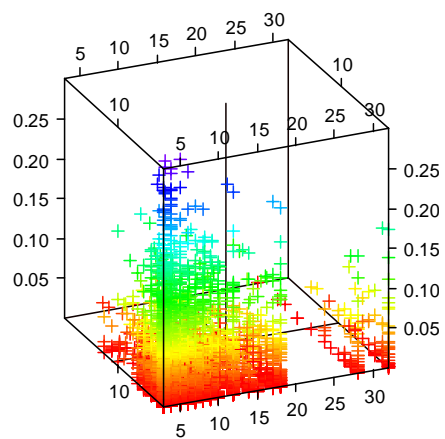

(a)

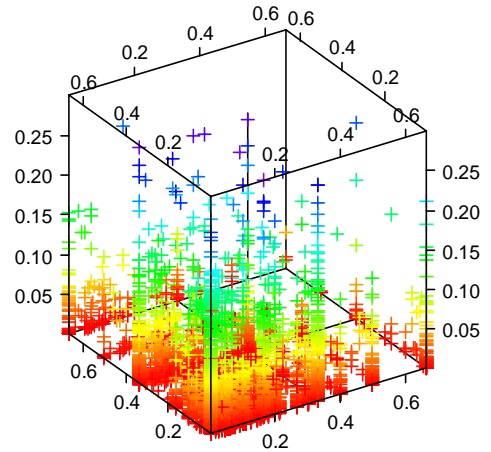

(b)



(c)

Figure 3: The increase of the algebraic connectivity $\mu^{*}$ (the $\mathrm{z}$ axis) due to the addition of link $\{i, j\}$ in relation with (a) the degree of node $i$ and $j$; (b) the clustering coefficient of node $i$ and $j$ (c) the betweenness of node $i$ and $j$ seperately in $\mathrm{x}$ and y axis in a BA model with $N=100$ and $m=3$.

in both the Erdös-Rényi random graph and the BA power law graph, we propose a topological metric based strategy that adds a link between a minimum degree node and a random other node, which are not originally connected. This strategy is also motivated by Theorem 3 and 4 , where the algebraic connectivity is shown to be limited by the lowest degree nodes. Furthermore, the metric degree is the simplest to calculate and it can be obtained only from the node and its neighbors, i.e. local information.

\subsection{Fiedler vector based strategy}

Maas [16] showed that after inserting an edge between node $i$ and $j$, the upper and lower bounds of the algebraic connectivity $\mu_{N-1}(G+e)$

$$
\begin{aligned}
& \min \left\{\mu_{N-1}(G)+\frac{\varepsilon \alpha^{2}}{\varepsilon+\left(2-\alpha^{2}\right)}, \mu_{N-2}(G)-\varepsilon\right\} \leq \\
& \mu_{N-1}(G+e) \leq \min \left\{\alpha^{2}+\mu_{N-1}(G), \mu_{N-2}(G)\right\}
\end{aligned}
$$

are related to $\alpha=\left|u_{i}-u_{j}\right|$, the absolute difference between the $i-t h$ and $j-t h$ elements of the Fiedler vector $u$ of $G$.
The Fiedler vector $u$ is the eigenvector corresponding to the second smallest eigenvalue (i.e., the algebraic connectivity) of the Laplacian matrix of a graph. In the lower bound, the first term increases with increasing $\varepsilon$ whereas the second one decreases. The highest lower bound can be achieved by a choice of $\varepsilon$ that makes both terms equal:

$$
\varepsilon=\frac{\beta-2}{2}+\left(\frac{(\beta-2)^{2}}{4}+\beta\left(2-\alpha^{2}\right)\right)^{\frac{1}{2}} \geq 0
$$

where $\beta=\mu_{N-2}-\mu_{N-1} \geq 0$. The higher $\alpha$ is, the lower is $\varepsilon$, and the higher the highest lower bound is. Higher $\alpha$ also contributes possibly to a higher upperbound. Hence, $\mu_{N-1}(G+e)$ tends to be large if $\alpha=\left|u_{i}-u_{j}\right|$ is large.

This is further illustrated by Figure 6, where all realizations of adding a link are performed in both an Erdös-Rényi random graph $G_{0.6}(100)$ and a BA power law graph with $N=100$ and $m=3$. The $\mu^{*}$ is positively correlated with $\alpha=\left|u_{i}-u_{j}\right|$.

Therefore, we propose the strategy of adding a link to the node pair $\{i, j\}$ with the highest $\alpha=\left|u_{i}-u_{j}\right|$, such that the algebraic connectivity can be increased the most. As shown 


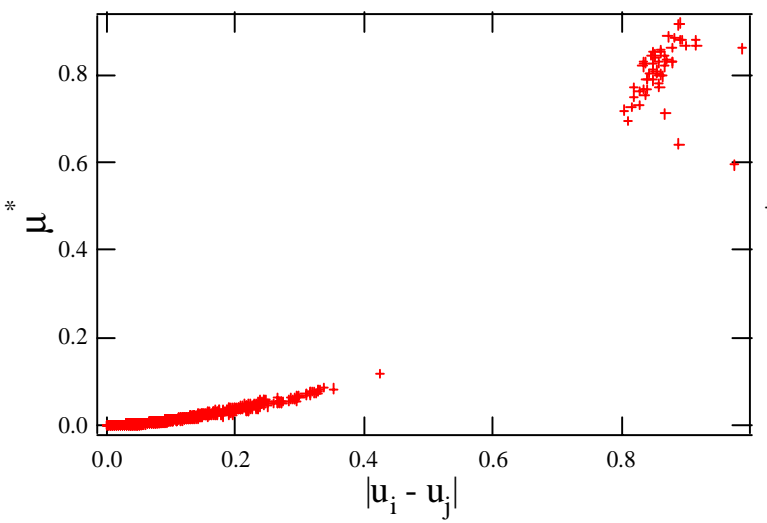

(a)



(b)

Figure 6: The increase of the algebraic connectivity $\mu^{*}$ due to the addition of link $\{i, j\}$ in relation with $\left|u_{i}-u_{j}\right|$ in $($ a) an Erdös-Rényi random graph $G_{0.6}(100)$ (b) a Barabási-Albert (BA) power law graph with $N=100$ and $m=3$.

in Figure 6, if we apply the strategy of adding a link to the node pair with the highest $\left|u_{i}-u_{j}\right|$, i.e. $\left|u_{i}-u_{j}\right|_{\max }=$ 0.986 and $\left|u_{i}-u_{j}\right|_{\max }=0.468$ in these two graphs, the algebraic connectivity is increased by $\mu^{*}=0.859$ and $\mu^{*}=$ 0.293 , which is close to the corresponding maximal possible increase of algebraic connectivity $\mu_{\max }^{*}=0.917$ and $\mu_{\max }^{*}=$ 0.301 , respectively.

\section{STRATEGY EVALUATION}

The strategies proposed are based on the correlation study in specific graphs. Since correlation between metrics are topology dependent, a strategy, that works in one topology, may not work in another network. Hence, in this section, we examine the validity of these two strategies in the class of the Erdös-Rényi random graphs, BA power law graphs and $k$-ary tree, where each node is connected to $k$ children. From the Erdös-Rényi random graph, over the BA model to the $k$-ary tree, both the randomness of node interconnections and link density decrease.

We carried out $10^{4}$ iterations for each simulation. In each iteration, an Erdös-Rényi random graph $G_{p}(N)$ or a BA power law graph is generated and the second and the third smallest Laplacian eigenvalues are calculated. First, we add a link between a random non-connected node pair without any strategy and obtain the normalized increase of the algebraic connectivity $\mu_{(0)}^{*}$. Second, we perform strategy 1 : add a link between the node with the minimum degree and a random non-connected node. The corresponding increase of the algebraic connectivity is $\mu_{(1)}^{*}$. Third, we perform strategy 2: add a link to the node pair $\{i, j\}$ with the highest $\alpha=\left|u_{i}-u_{j}\right|$ and obtain $\mu_{(2)}^{*}$.

\subsection{Erdös-Rényi random graph $G_{p}(N)$}

Simulations are carried out for Erdös-Rényi random graph with $p=0.6, N=50,100,200,400,800$. Besides, with fixed $N=200$, we perform simulations for $p=0.2,0.4,0.6$ and 0.8 . We evaluate the gain of the strategy by comparing with the link addition without any strategy, i.e. $\operatorname{Pr}\left[\mu_{(s)}^{*}-\mu_{(0)}^{*} \geq\right.$ $x], s=1$ and 2 for strategy 1 and 2, respectively. Any link addition, including the optimal one follows $\mu^{*} \leq 1$. Hence, $\mu_{(s)}^{*}-\mu_{(0)}^{*}<1$. Figure 7 and 8 show that both

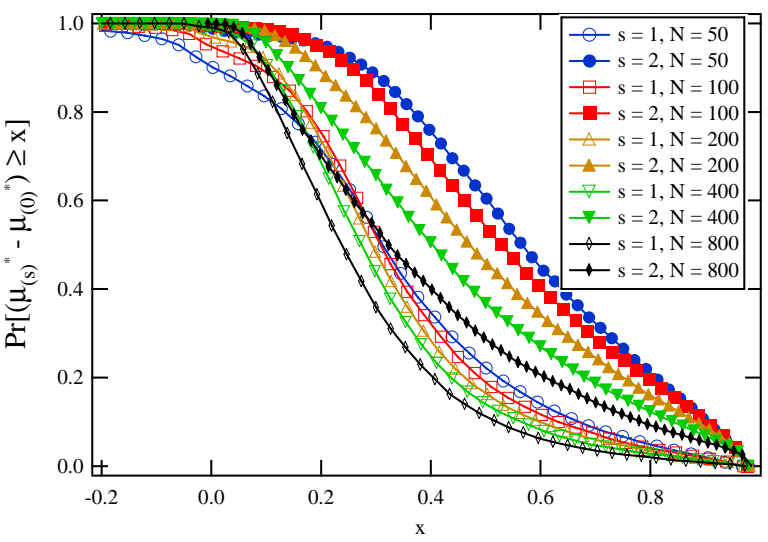

Figure 7: Gain $\operatorname{Pr}\left[\mu_{(s)}^{*}-\mu_{(0)}^{*} \geq x\right]$ of stragtegy $1(s=1)$ and strategy $2(s=2)$ in the Erdös-Rényi random graph $G_{p}(N)$, where $p=0.6$.

strategies perform better than random link addition, with probability larger than 0.9 , i.e. $\operatorname{Pr}\left[\mu_{(s)}^{*}-\mu_{(0)}^{*} \geq 0\right]>0.9$. Both strategies work better for smaller networks as shown in Figure 7. Strategy 2 performs better than strategy 1 in Erdös-Rényi random graphs with various network size $N$ and link density $p$. The effect of the link density is not obvious in Erdös-Rényi random graphs which are generally dense. As shown in Table 1 , the effect of strategy 1 and 2 is evident in $E\left[\mu_{(1)}^{*}\right]$ and $E\left[\mu_{(2)}^{*}\right]$ compared to $E\left[\mu_{(0)}^{*}\right]$ when no strategy is applied. Strategy 2 performs better, but it requires the knowledge of the topology of the whole network. Only local information, the degree, is needed in strategy 1.

\subsection{BA model}

The same simulations are carried out in the BA model with $N=100,200,400$ and $m=3,4,5$. As illustrated in Figure 9 , strategy 1 behaves the same as link addition without strategy, while, strategy 2 performs better with probability round 0.9 than random link addition, i.e. $\operatorname{Pr}\left[\mu_{(s)}^{*} \geq \mu_{(0)}^{*}\right] \approx$ 
Table 1: Strategies comparison in Erdös-Rényi random graphs.

\begin{tabular}{|c|c|c|c|c|c|}
\hline$p=0.6$ & $N=50$ & $N=100$ & $N=200$ & $N=400$ & $N=800$ \\
\hline$E\left[\mu_{(2)}^{*}\right]$ & 0.644 & 0.594 & 0.532 & 0.469 & 0.401 \\
\hline$E\left[\mu_{(1)}^{*}\right]$ & 0.407 & 0.387 & 0.364 & 0.340 & 0.303 \\
\hline$E\left[\mu_{(0)}^{*}\right]$ & 0.049 & 0.0214 & 0.00951 & 0.0042 & 0.00169 \\
\hline$N=200$ & $p=0.2$ & $p=0.4$ & $p=0.6$ & $p=0.8$ & \\
\hline$E\left[\mu_{(2)}^{*}\right]$ & 0.588 & 0.544 & 0.532 & 0.579 & \\
\hline$E\left[\mu_{(1)}^{*}\right]$ & 0.481 & 0.396 & 0.364 & 0.391 & \\
\hline$E\left[\mu_{(0)}^{*}\right]$ & 0.012 & 0.0092 & 0.0095 & 0.011 & \\
\hline
\end{tabular}

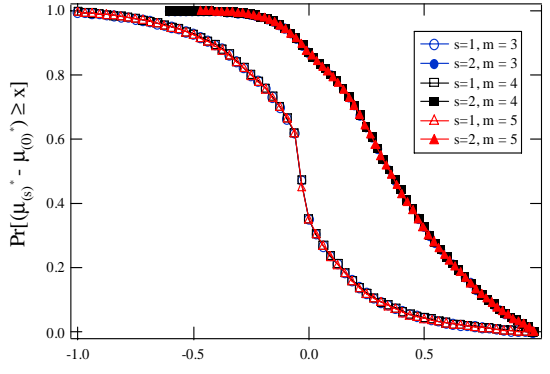

(a)

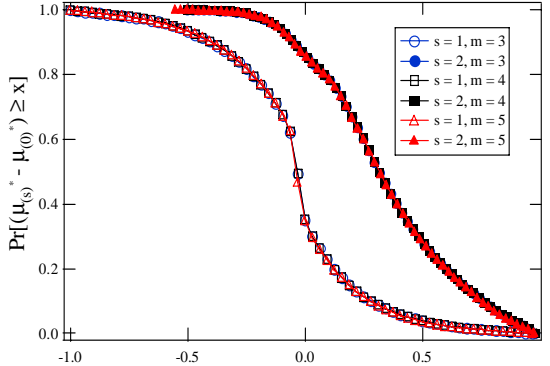

(b)

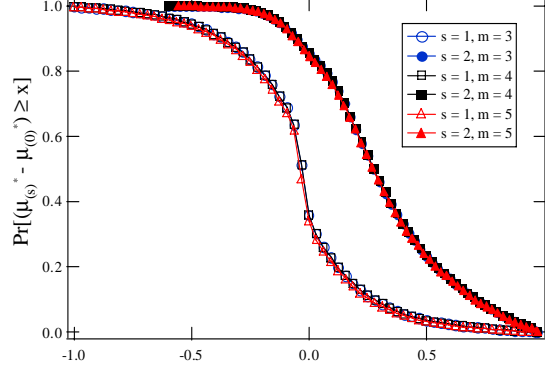

(c)

Figure 9: Gain $\operatorname{Pr}\left[\mu_{(s)}^{*}-\mu_{(0)}^{*} \geq x\right]$ of stragtegy $1(s=1)$ and strategy $2(s=2)$ in the BA model with (a) $N=100$ (b) $N=200$ and (c) $N=400$.

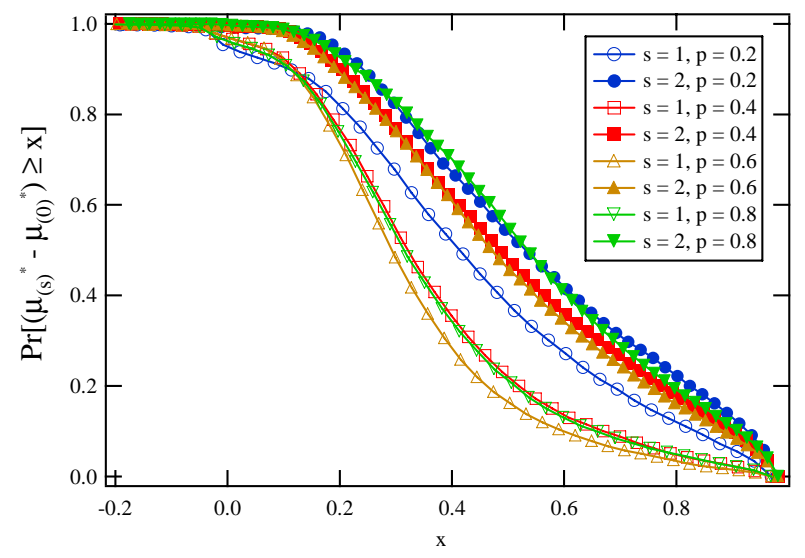

Figure 8: Gain $\operatorname{Pr}\left[\mu_{(s)}^{*}-\mu_{(0)}^{*} \geq x\right]$ of stragtegy $1(s=1)$ and strategy $2(s=2)$ in the Erdös-Rényi random graph $G_{p}(N)$, where $N=200$.
0.9. Furthermore, $m$ (or equivalently the link density) has no effect on the performance of both strategies. Figure 10 shows that strategy 2 works slightly better on BA power law graphs with smaller size $N$.

Table 2: Strategies comparison in BA power law graphs.

\begin{tabular}{|c|c|c|c|}
\hline$m=3$ & $N=100$ & $N=200$ & $N=400$ \\
\hline$E\left[\mu_{(2)}^{*}\right]$ & 0.558 & 0.521 & 0.477 \\
\hline$E\left[\mu_{(1)}^{*}\right]$ & 0.153 & 0.146 & 0.142 \\
\hline$E\left[\mu_{(0)}^{*}\right]$ & 0.170 & 0.161 & 0.148 \\
\hline
\end{tabular}

\section{3 $\quad K$-ary tree}

We investigate the $k$-ary tree [17] of $\operatorname{depth}^{2} D$ where each node has exactly $k$ children. In a $k$-ary tree the total number of nodes is

$$
N(D)=1+k+k^{2}+\cdots+k^{D}=\left\{\begin{array}{l}
\frac{k^{D+1}-1}{k-1}, \quad k \neq 1 \\
1+D, \quad k=1
\end{array}\right.
$$

Since the interconnections of nodes in a $k$-ary tree is determined, we examine one specific $k$-ary tree with $k=2, d=7$ and try all the possibilities of adding a link. On average, the algebraic connectivity can be increased by $E\left[\mu_{(0)}^{*}\right]=0.137$. On average, strategy 1 improves the algebraic connectivity by $E\left[\mu_{(1)}^{*}\right]=0.129<E\left[\mu_{(0)}^{*}\right]$, which is not better than random link addition. Given the level of the node pair, where

${ }^{2}$ The depth $D$ is the number of hops (or links) from the root to a node at the leaves. 


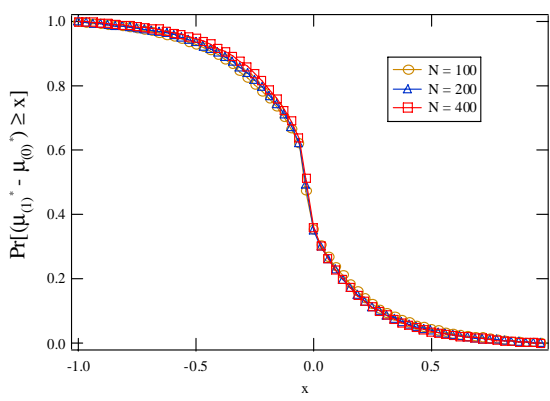

(a)



(b)

Figure 10: (a) Gain $\operatorname{Pr}\left[\mu_{(1)}^{*}-\mu_{(0)}^{*} \geq x\right]$ of stragtegy 1 and (b) gain $\operatorname{Pr}\left[\mu_{(2)}^{*}-\mu_{(0)}^{*} \geq x\right]$ of strategy 2 in the BA model with $m=3$.

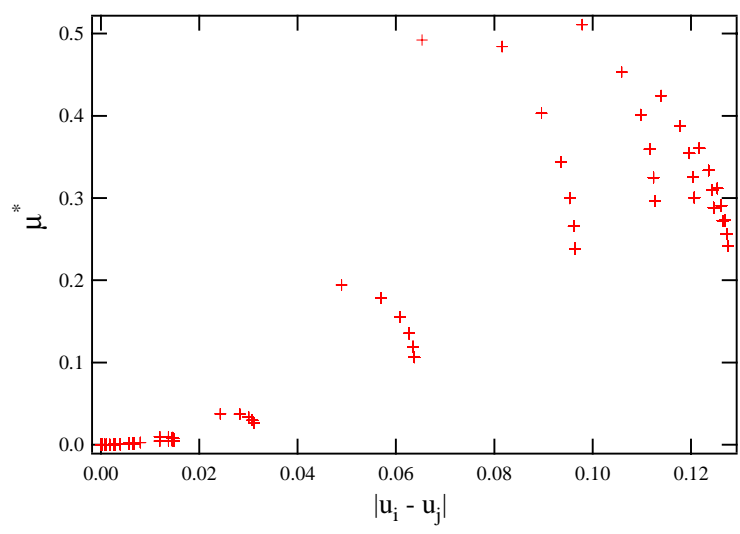

Figure 11: The increase of the algebraic connectivity $\mu^{*}$ due to the addition of link $\{i, j\}$ in relation with $\left|u_{i}-u_{j}\right|$ in a $k$-ary tree with $k=2, d=7$.

a link is added, $\mu^{*}$ is found to be higher if these two nodes share fewer common parents. The optimal link addition is between two nodes both in level 2. Hence, none of them has degree 1. As shown in Figure 11, the positive correlation between $\mu^{*}$ and $\left|u_{i}-u_{j}\right|$ still remains, but weaker than in the Erdös-Rényi random graph and the BA power law graph. The increase of algebraic connectivity achieved by strategy 2 is $\mu_{(2)}^{*}=0.24$, round half of the maximal possible increase $\mu_{\text {max }}^{*}=0.51$.

\subsection{Comparison with optimal link addition}

Our strategies are evaluated via $\operatorname{Pr}\left[\mu_{(s)}^{*}-\mu_{(0)}^{*} \geq x\right], s=1$ and 2 for strategy 1 and 2 respectively, by comparing them with the random link addition. One may be curious to know how far our strategies are from the optimal link addition. The maximal possible increase or optimal increase of the algebraic connectivity $\mu_{\max }^{*}$ can be obtained by trying all possible ways of adding a link to a network. Due to the computational complexity, we compare our strategies with the optimal link addition in $10^{4}$ Erdös-Rényi random graphs $G_{0.6}(100)$ and in $10^{4}$ BA power law graphs with $N=100,200$ and $m=5$. As shown in Table 3, strategy 2 performs generally better than strategy 1 , and is close to the optimal link addition, especially in Erdös-Rényi random
Table 3: Strategies comparison with optimal link addition

\begin{tabular}{|c|c|c|c|}
\hline & $G_{0.6}(100)$ & $\begin{array}{l}B A \text { model } \\
N=100, m=5\end{array}$ & $\begin{array}{l}B A \text { model } \\
N=200, m=5\end{array}$ \\
\hline$E\left[\mu_{\max }^{*}\right]$ & 0.62 & 0.87 & 0.88 \\
\hline$E\left[\mu_{(2)}^{*}\right]$ & 0.59 & 0.56 & 0.52 \\
\hline$E\left[\mu_{(1)}^{*}\right]$ & 0.39 & 0.15 & 0.14 \\
\hline$E\left[\mu_{(0)}^{*}\right]$ & 0.021 & 0.17 & 0.16 \\
\hline
\end{tabular}

graphs.

\section{CONCLUSION}

The difficulty of applying strategies to optimize the algebraic connectivity (or in general a topological metric $R$ ) by adding a link $e=(i, j)$ based on topological characteristics of node pair $\{i, j\}$ is due to the fact that the correlation between metrics (e.g. between $\mu^{*}$ and degrees of the node pair) is topology dependent. In other words, a strategy, that works for a given type of graphs, may not work in other classes of networks.

Strategy 1 seems to perform better in the class of dense graphs such as the Erdös-Rényi random graphs, although the effect of the specific link density of Erdös-Rényi random graph is not obvious. However, strategy 1 looses its effect in sparse networks like the BA power law graph and the $k$-ary tree, where many nodes possess the minimal degree. Another extreme example is $G=K_{N} \backslash\{i, j\}$, the complete graph except one link. Each node has the maximal degree $N-1$ except that node $i$ and $j$ have the minimal degree $N-2$. Adding a link between $i$ and $j$ leads to the maximal increase of the algebraic connectivity $\mu_{N-1}(G+e)=\mu_{N-1}(G)+2$ or $\mu^{*}=1$ according to Theorem 2 .

Strategy 2 seems to be applied better in graphs with more randomness in interconnections of nodes. From the ErdösRényi random graph, over the BA model to the $k$-ary tree, the randomness of node interconnections decreases. Strategy 2 performs better in Erdös-Rényi random graphs and BA power law graphs than in the $k$-ary tree, which is regular in node interconnections. In other words, the positive correlation between $\mu^{*}$ and $\left|u_{i}-u_{j}\right|$ is weaker in the $k$-ary tree. The worst case is the regular D-lattice, where each node has $2 \mathrm{D}$ neighbors. A D-lattice is even more regular 
than a $k$-ary tree in the sense that more nodes have the same degree except nodes in the border. The second and third smallest eigenvalue of a D-lattice are the same. The algebraic connectivity remains the same wherever a link is added. Hence, the correlation between $\mu^{*}$ and $\left|u_{i}-u_{j}\right|$ disappears completely.

Strategy 2 performs generally better than strategy 1 in Erdös-Rényi random graph, BA model and $k$-ary tree. However, strategy 2 requires the Fiedler vector, or the whole network topology, while only the node degree is needed in strategy 1, which can be applied even when the network is partially known. The computational complexity ${ }^{3}$ is reduced from $O\left(N^{5}\right)$ of the optimal link addition searching to $O\left(N^{3}\right)$ by strategy 2 and to $O(N)$ by strategy 1 . Finally, strategy 2 is shown to be close to the optimal link addition, especially in Erdös-Rényi random graphs.

\section{ACKNOWLEDGEMENT}

We would like to thank J. Martín Hernández for the help in our simulations. This research was supported by the Netherlands Organization for Scientific Research (NWO) under project number 643.000.503.

\section{REFERENCES}

[1] N.M. Luscombe et al., "Genomic analysis of regulatory network dynamics reveals large topological changes", Nature 431, p. 308 (2004).

[2] H. Jeong et al., "The large-scale organization of metabolic networks", Nature 407, p. 651 (2000).

[3] A.-L Barabasi, Linked, The new science of networks, Perseus, Cambridge, MA, 2002.

[4] R. Albert, A. Barabasi, Science 286, 509-512, 1999.

[5] K. Takemotoa and C. Oosawa, "Modeling for evolving biological networks with scale-free connectivity, hierarchical modularity, and disassortativity", Mathematical Biosciences 208, 454-468 (2007).
[6] L. Donetti, F. Neri and M. A. Muñoz, Optimal network topologies: Expanders, Cages, Ramanujan graphs, Entangled networks and all that, J. Stat. Mech. (2006) P08007.

[7] Wang X F and Chen G, Synchronization in small-world dynamical networks, Int. J. Bifurcation Chaos Appl. Sci. Eng. 12 187, 2002.

[8] Wang X F and Chen G, Synchronization in scale-free dynamical networks: robustness and fragility, IEEE Trans. Circ. Syst. I 49 54, 2002.

[9] B. Mohar, Y. Alavi, G. Chartrand, O.R. Oellermann and A.J. Chwenk: The Laplacian spectrum of graphs, Graph Theory, Combinatorics and Applications 2, pp. 871-898 (1991).

[10] M. Fiedler, Algebraic connectivity of graphs, Czechoslovak Math. J. 23:298-305 (1973).

[11] Y. Fan, On spectral integral variations of graphs, Linear and Multilinear Algebra 50 (2002) 133-142.

[12] S. Barik, S. Pati, On algebraic connectivity and spectral integral variations of graphs, Linear Algebra Appl. 397 (2005) 209-222.

[13] K.Ch. Das, The Laplacian spectrum of a graph, Computers \& Mathematics with Applications, Volume 48, Issues 5-6, September 2004, Pages 715-724.

[14] M. E. J. Newman, Phys. Rev. E 64, 016131 (2001).

[15] M. Faloutsos, P. Faloutsos, and C. Faloutsos, "On power law relationships of the Internet topology," Proc. ACM SIGCOMM 1999, ACM Comput. Commun. Rev., vol. 29, pp. 251-262, 1999.

[16] C. Maas, Transportation in graphs and the admittance spectrum, Discrete Applied Mathematics, v.16 n.1, p.31-49, Jan. 1987.

[17] P. Van Mieghem, Performance Analysis of Communications Systems and Networks, Cambridge University Press, 2006.

\footnotetext{
${ }^{3}$ The computational complexity of eigenvalues is $O\left(N^{3}\right)$.
} 